\title{
A strategic capacity allocation model for a complex supply chain: Formulation and solution approach comparison
}

\author{
Hongyan Li*, Linda Hendry, Ruud Teunter \\ Management Science Department, Lancaster University Management School, Lancaster LA1 4YX, UK
}

Received 12 April 2006; accepted 9 February 2007

Available online 24 March 2007

\begin{abstract}
In this paper, a capacity allocation problem is discussed based on a more complex supply chain than has been typically considered in previous quantitative modelling studies. This study analyses an integrated supply chain operation from raw material purchasing to final product distribution. The aim is to optimize the allocation of capacities among different facilities and product items. In this paper, a mixed integer programming model with dynamic characteristics is presented first, and then alternative solution procedures are introduced. The solution procedures include the development of a decomposition heuristic and an integrated heuristic algorithm. A computation study compares the solution procedures and uses sensitivity analysis to show that the heuristics work well. Thus, by adequately modelling a more realistic sized supply chain problem, this study represents an important advance in supply chain modelling research.
\end{abstract}

(C) 2007 Elsevier B.V. All rights reserved.

Keywords: Supply chain management; Supply chain modelling and optimization; Strategic capacity allocation; Large-scale mixed integer programming; Heuristics

\section{Introduction}

Quantitative modelling for strategic supply chain planning continues to be a fruitful research area. The purpose of the modelling is usually to provide effective decision support for strategic resource allocation in the longer term, including factors such as: Selection of suppliers, configuration of manufacturers and distributors' capacities, as well as allocation of these capacities to products and so forth. At present, increased world competition is forcing supply chain companies to reconsider their capacity allocation strategies, particularly given that

\footnotetext{
*Corresponding author.

E-mail address: h.li7@lancaster.ac.uk (H. Li).
}

decisions, such as capacity allocation decisions, have a significant impact on supply chain performance. Given limited raw material supplies and limited capacities for production, final product transportation and distribution centres, the capacity allocation problem determines how best to use these resources to meet final product demand.

Although quite a few researchers have done valuable studies regarding strategic supply chain capacity allocation problems, the majority of the published research treats each stage of the supply chain as a separate system, e.g. only the manufacturing stage, or production and distribution integration. Few studies have considered a supply chain network from raw material procurement to final product distribution and their interactions. 
However, as Park (2005) said "in order to implement the integrated planning approach properly, a company must involve all major planning operations in model construction and data processing". Thus by including raw material procurement and final product distribution in the model, this study addresses a more realistic supply chain problem than those typically previously discussed in the literature. In addition, this paper considers the problem of how to allocate capacity over time rather than looking at a single planning period. This is a significant advance over previous approaches that have considered the raw material purchasing stage which have focused on a single period, making significant simplifying assumptions.

The importance of considering a more realistic supply chain problem has been shown by researchers such as Park (2005) who discussed the effectiveness of integrated analysis for a production and distribution planning problem. He found that the integrated planning approach produced more net profit than the decoupled method for supply chain planning, and produced a higher demand fill rate. In other words, the integrated planning methods were shown to lead to better solutions.

In this paper, a strategic capacity allocation problem for a complex supply chain operation process is considered with a series of fixed operation costs and supply, production, transportation, and distribution capacity constraints. First, a large-scale mixed integer programming (MIP) model is formulated to solve a multi-period, multi-product capacity allocation problem involving multiple suppliers, multiple production sites and multiple distribution centres. The model objective is to maximize the overall profit of the whole supply chain, and to minimize the costs of raw material purchasing and inventory, product production, transportation and distribution, as well as inventory holding and product shortage, production changeover, etc. In addition, the model also considers the dynamic characteristics of production and inventories in different stages of the supply chain.

Given that MIP can only be solved for small problems due to computational time restrictions, heuristics are considered to solve the large problems and two alternative algorithms are compared. The sensitivity of the capacity and price parameters to the relative performance of the algorithms is also addressed.

The remainder of this paper is organized as follows. Section 2 presents a review of related literature and further explains the advances in the approach proposed. In Section 3, the proposed problem is specified and the mathematical model is presented. Sections 4 and 5 describe the algorithms and show the computational results. Sensitivity analysis results are reported in Section 6. Section 7 presents some results on a comparison study with Park's study. Finally, conclusions and future research directions are given in Section 8.

\section{Literature review}

Many researchers have made tremendous contributions to capacity allocation methods, but the majority of the published articles on capacity allocation have focused on the single-period problem, or the manufacturing stage rather than the entire supply chain network. For example, Eppen et al. (1989) presented a multi-product, multi-plant, multi-period capacity allocation model. It was probably one of the earliest successful efforts to model capacity allocation problems. The authors presented a stochastic MIP model based on a scenario planning approach. In this approach, the authors assumed that the demand was realized before the production decision is made, and no inventory is held from one period to the next. Although this approach has generated a practical application model, it was limited to a single manufacturing stage.

To overcome the limitations of considering only a single manufacturing stage, a number of researchers have considered the integrated production-distribution problem. Vidal and Goetschackx (1997) presented a critical extensive literature review of strategic production-distribution models. In this paper, the authors categorized the literature into four groups: Previous reviews, optimization models, additional issues for modelling, and case studies. A particular emphasis of their review was on MIP models. Thus, they identified the main characteristics of the MIP models, including the terms considered in the objective function, the constraints, and the specific characteristics of the solution methods and computation experiences.

Since this important review, further examples of studies addressing the production-distribution problem have emerged. For example, Dhaenens-Flipo and Finke (2001) studied a deterministic MIP model considering multi-products with significant set-ups, multi-facilities, and multi-periods simultaneously. The authors implemented a number of numerical 
experiments with different sizes of problems. Although this study discussed the interactions between production and distribution activities, the final model is based on some strict assumptions such as no transportation and distribution capacity constrains and so forth. As with all models of this type, the key limitation is that the raw material procurement activities are not considered.

More recently, Ryu et al. (2004) addressed bilevel decision-making problems in the context of enterprise-wide supply chain optimization with one level corresponding to a plant planning problem, and the other one corresponding to a distribution network problem. This study explored some issues on decomposition of supply chain planning problems. Although the authors intend to consider production and distribution activities simultaneously, they just proposed a decoupled linear programming model, rather than realizing the integration of the supply chain. Moreover, the raw material procurement procedure is not combined into their model. In addition, the authors considered a single decision making objective, minimization of overall cost. This is insufficient at present given the increase of competition in the market which has led to the need to include other factors in the objective such as the demand fill rate or customer service level. In general, this model was significantly oversimplified since all fixed cost factors and logical constraints are ignored.

In a recent paper on supply chain integrated modelling, Park (2005) presented a method for integrated production and distribution planning. He investigated the effectiveness of the integration through a computational study with the objective of maximizing the total net profit. This is considered one of the best production-distribution models in the literature, because it is a relatively realistic model considering multiple capacity constraints within a multi-period planning horizon. Moreover, the model involves some fixed costs at different operation stages. Having proposed a MIP model, Park then presented alternative solutions and compared them in a computational study. In addition, sensitivity analysis was carried out on capacities and fixed costs. However, this study assumed that the plants have unlimited storage capacity and the firm can change the fleet size freely without extra cost, but in real operations, these assumptions are not often realized. Additionally, the model did not take changeover cost and production batch size constraints into account at the production stage. Moreover, the solution procedures have some limitations, for example, the decoupled models do not always give feasible solutions since they ignore the interactions of different operation stages. Although the problem considered a supply chain network configuration including multi-plants, multi-retailers, multi-items, and multi-period environment, the key disadvantage is that no raw material procurement activities were considered.

Whilst there have been a considerable number of papers that consider the production-distribution problem, fewer studies have added the integration of the raw material procurement. Three key exceptions are discussed next. Firstly, Jayaraman and Pirkul (2001) presented a MIP model in which they noted the raw material input issue to manufacturing plants with traditional production transportation and distribution together. However, this is a singleperiod model without consideration of set-up times or costs and changeover costs. In addition, this paper presented a heuristic algorithm based on Lagrangean relaxation. The influence of capacity constraints on the solution was not addressed.

Additionally, Lim and Kim (1999) and Chen and Wang (1997) both studied the integrated production and distribution planning problem considering raw material purchasing. Although the authors intended to solve a relatively practical integrated supply chain planning problem, the model was oversimplified. First, they both formulated the problem as a single-period linear programming model; second, their linear programming methods ignored some key fixed costs; furthermore, raw material and product inventory are not considered.

In conclusion, whilst there have been some attempts to consider the integrated supply-production-distribution problem in the literature, all of these have considerable simplifying assumptions. Thus, the addition of the raw material procurement activities has not led to an overall improvement in the realism of the supply chain considered. Therefore, the model of Park is considered to be the most realistic supply chain model in the literature to date, even though it does not include the raw material procurement activities. In the model presented in Section 3 below, the major influence is the realism attained by Park as it assumes a multi-period, multiplant, multi-retailer and multi-item environment. It then makes advances on this model by adding in the integration of the supplier raw material procurement problem; and making more realistic 
assumptions regarding storage capacity restrictions and fleet size, and including changeover costs. In terms of solution methods, heuristic algorithms and decomposition algorithms have been studied and applied extensively in the literatures. In this study, similar approaches are attempted to those discussed in Park (2005).

\section{Model formulations}

\subsection{Problem description}

In this paper, a supply chain network based on raw material flow and product flow as depicted in Fig. 1 will be considered.

This complex supply chain network includes multiple plants, distributions centres and suppliers. Based on the network, a hypothetical capacity allocation problem will be considered, where multiple products can be produced within a planning horizon of 3-5 years. The aim is to determine how capacities should be allocated optimally to product items in a complete supply chain, whereby the capacity constraints of supply, production, transportation and distribution are considered simultaneously. Here, production capacity is defined as the available production time in each plant and each period, and the capacity of each plant is independent of the others; distribution and transportation capacity are the maximum volume available in each transportation fleet and distribution site in each period, and the supply capacity is the maximum amount of raw material that can be provided by each supplier in each period. In addition, some other factors, such as production changeovers and minimum production batch size limitations, are considered. Overall, the study is based on the following assumptions:

(1) No inventory is held in production sites for all periods. In other words, all products are transported to distribution centres in each

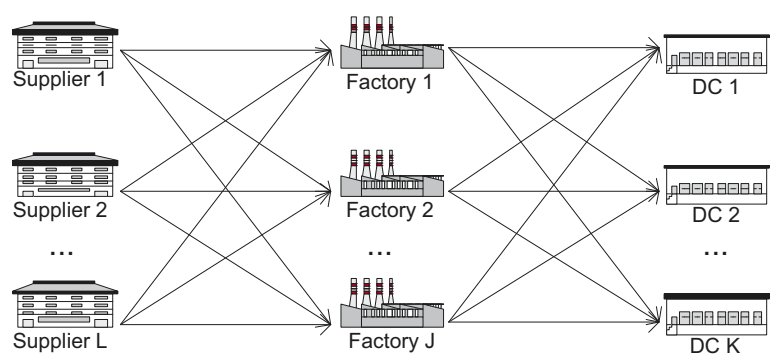

Fig. 1. Supply chain network structure. period, and product inventory incurs in distribution sites at the end of each period.

(2) The model considers the demands generated at each distribution centre independently from each other.

(3) A type of product can be produced in more than one plant, and each plant can produce at least one type of product.

(4) There is transportation between certain production sites and distribution centres, but no transportation between production sites.

(5) The model uses some big time bucket formulations, namely, quarterly periods are considered.

(6) The plants usually hold raw material stock to maintain production.

(7) The time lags between procurement, production and distribution are assumed to be negligible. This is reasonable if the time bucket is large compared to those time lags. In our numerical experiment (Section 5), the time unit is set to 3 months.

\subsection{Mathematical model}

The notations that will be used to describe the problem and algorithm are as follows:

(1) Indices:

$i$, index on product, where $i=1, \ldots, I, I$ is the number of types of products produced;

$j$, index on plant, where $j=1, \ldots, J, J$ is the number of plants (production sites);

$k$, index on distribution centre, where $k=1, \ldots$, $K, K$ is the number of distribution centres;

$l$, index on supplier, where $l=1, \ldots, L, L$ is the number of suppliers;

$h$, index on raw material, where $h=1, \ldots, H, H$ is the number of types of raw materials;

$t$, index on periods of planning, where $t=1, \ldots$, $T$ or $t=1, \ldots, n, n \leqslant T$.

(2) Demand parameters:

$d_{i k t}$, demand for product $i$ generated from distributor $k$ in period $t$.

(3) Capacity parameters:

$\mathrm{CS}_{l h t}$, capacity of supplier $l$ for raw material $h$ in period $t$ (amount of raw material);

$\mathrm{CP}_{j t}$, production capacity of plant $j$ in period $t$ (available production time);

$\mathrm{CD}_{k t}$, capacity of distribution centre $k$ in period $t$ (available inventory space);

$\mathrm{CT}_{j t}$, transportation capacity from plant $j$ in period $t$ (the number of lorries). 
(4) Cost parameters:

$\operatorname{cfv}_{\text {lhjt }}$, fixed procurement cost of raw material $h$ from supplier $l$ delivered to plant $j$ in period $t$; $\mathrm{cvv}_{l h j t}$, variable procurement cost per unit of raw material $h$ from supplier $l$ delivered to plant $j$ in period $t$;

$\mathrm{cfp}_{i j t}$, fixed production cost in plant $j$ for product $i$ in period $t$;

$\mathrm{cvp}_{i j t}$, variable production cost in plant $j$ per unit of product $i$ in period $t$;

$\mathrm{cft}_{i j k t}$, fixed cost of transporting product $i$ from plant $j$ to distribution centre $k$ in period $t$;

$\mathrm{cvt}_{i j k t}$, variable cost of transportation per unit of product $i$ from plant $j$ to distribution centre $k$ in period $t$;

$\mathrm{cfs}_{i k t}$, fixed distribution centre operations cost of product $i$ in distribution centre $k$ in period $t$;

cvs $_{i k t}$, variable distribution centre operations cost for per unit of product $i$ in distribution centre $k$ in period $t$;

$\mathrm{cco}_{j t}$, average changeover cost of plant $j$ in period $t$ between two sequential setups;

$\operatorname{civ}_{i k t}$, holding costs of product $i$ in distribution centre $k$ at the end of period $t$;

$\mathrm{cs}_{i t}$, shortage cost of product $i$ in period $t$;

$\mathrm{rmsc}_{h j t}$, inventory cost of raw material $h$ in plant $j$ in period $t$.

(5) Other parameters:

$r_{i h}$, amount of raw material $h$ needed to produce one unit of product $i$;

$\mathrm{pt}_{i j}$, processing time per unit of product $i$ in plant $j$;

sut $_{i j}$, set-up time of product $i$ in plant $j$;

ups $_{i k}$, fraction of distribution capacity required for distributing product $i$ in distribution centre $k$;

$\mathrm{PLC}_{i}$, the capacity of each lorry for product $i$;

$\mathrm{MBP}_{i}$, the minimum production batch size of product $i$;

$p_{i t}$, the price of product $i$ in period $t$;

$G_{i j t}^{x}, G_{i j k t}^{y}, G_{i k t}^{u}$ are large numbers greater than

total demand, and $G_{l h j t}^{z}$ is a big number greater than $r_{i h}$ times total demand.

(6) Decision variables:

$x_{i j t}$, amount of product $i$ produced in plant $j$ in period $t$;

$y_{i j k t}$, amount of product $i$ delivered to distribution centre $k$ from plant $j$ in period $t$;

$u_{i k t}$, amount of product $i$ distributed by distribution centre $k$ in period $t$;

$z_{l j h t}$, amount of the raw material $h$ supplied to plant $j$ from supplier $l$ in period $t$; $\tau_{i k t}$, unsatisfied demand for product $i$ produced from distribution centre $k$ in period $t$; $\mathrm{rm}_{h j t}$, the inventory amount of raw material $h$ in plant $j$ in period $t$;

$$
\begin{gathered}
X_{i j t}= \begin{cases}1 & \text { if } x_{i j t}>0, \\
0 & \text { if } x_{i j t}=0,\end{cases} \\
U_{i k t}= \begin{cases}1 & \text { if } u_{i k t}>0, \\
0 & \text { if } u_{i k t}=0,\end{cases} \\
Y_{i j k t}=\left\{\begin{array}{ll}
1 & \text { if } y_{i j k t}>0, \\
0 & \text { if } y_{i j k t}=0,
\end{array} ;\right. \\
Z_{l h j t}= \begin{cases}1 & \text { if } z_{l h j t}>0, \\
0 & \text { if } z_{l h j t}=0,\end{cases}
\end{gathered}
$$

$I_{i k t}$, inventory amount of product $i$ in distribution centre $k$ at the end of period $t$;

$\zeta_{i j}$, a general integer to represent the number of changeovers.

With respect to the problem defined above, a MIP model $\mathrm{P}$ is formulated

$$
\begin{aligned}
P: \max V= & \sum_{t=1}^{T}\left(\sum_{i=1}^{I} p_{i t} \sum_{k=1}^{K}\left(d_{i k t}-\tau_{i k t}\right)\right. \\
& -\left(\sum_{i=1}^{I} \sum_{j=1}^{J} \mathrm{cvp}_{i j t} x_{i j t}+\sum_{i=1}^{I} \sum_{j=1}^{J} \mathrm{cfp}_{i j t} X_{i j t}\right. \\
& +\sum_{i=1}^{I} \sum_{j=1}^{J} \sum_{k=1}^{K} \mathrm{cvt}_{i j k t} y_{i j k t} \\
& +\sum_{i=1}^{I} \sum_{j=1}^{J} \sum_{k=1}^{K} \mathrm{cft}_{i j k t} Y_{i j k t} \\
& +\sum_{i=1}^{I} \sum_{k=1}^{K} \mathrm{cvs}_{i k t} u_{i k t}+\sum_{i=1}^{I} \sum_{k=1}^{K} \mathrm{cfs}_{i k t} U_{i k t} \\
& +\sum_{j=1}^{J} \mathrm{cco}_{j t} \zeta_{j t}+\sum_{j=1}^{J} \sum_{i=1}^{I} \sum_{k=1}^{K} \mathrm{civ}_{i k t} I_{i k t} \\
& +\sum_{i=1}^{I} \mathrm{cs}_{i t} \sum_{k=1}^{K} \tau_{i k t} \\
& +\sum_{l=1}^{L} \sum_{h=1}^{H} \sum_{j=1}^{J} \operatorname{cvv}_{l h j t} z_{l h j t} \\
& +\sum_{l=1}^{L} \sum_{h=1}^{H} \sum_{j=1}^{J} \operatorname{cfv}_{l h j t} Z_{l h j t} \\
& \left.\left.+\sum_{h=1}^{H} \sum_{j=1}^{J} \operatorname{rmsc}_{h j t} \mathrm{rm}_{h j t}\right)\right)
\end{aligned}
$$


Subject to

Demand constraints:

$\sum_{t=1}^{n} u_{i k t} \geqslant \sum_{t=1}^{n} d_{i k t} \times 0.95, \quad \forall i, k, \quad n=1, \ldots, T$,

$u_{i k t}=d_{i k t}+I_{i k t}-I_{i k(t-1)}-\tau_{i k t}, \quad \forall i, k, t$,

$I_{i k 0}=0, \quad \forall i, k$.

Logical constraints:

$\sum_{k=1}^{K} y_{i j k t}=x_{i j t}, \quad \forall i, j, t$,

$\sum_{j=1}^{J} y_{i j k t}=u_{i k t}, \quad \forall i, k, t$,

$\sum_{t=1}^{n} \sum_{l=1}^{L} z_{l h j t} \geqslant \sum_{t=1}^{n} \sum_{i=1}^{I} x_{i j t} r_{i h}, \quad \forall h, j, \quad n=1, \ldots, T$,

$\sum_{l=1}^{L} z_{l h j t}+\operatorname{rm}_{h j(t-1)} \geqslant \sum_{i=1}^{I} r_{i h} x_{i j t}+\operatorname{rm}_{h j t}, \quad \forall h, j, t$,

$\operatorname{rm}_{h j 0}=0, \quad \forall h, j$.

Production batch size constraints:

$x_{i j t} \geqslant \mathrm{MBP}_{i} \times X_{i j t}, \quad \forall i, j, t$.

Capacity constraints:

$\sum_{i=1}^{I}\left(p t_{i j} x_{i j t}+\operatorname{sut}_{i j} X_{i j t}\right) \leqslant \mathrm{CP}_{j t}, \quad \forall j, t$,

$\sum_{i=1}^{I} u_{i k t} \times \operatorname{ups}_{i k} \leqslant \mathrm{CD}_{k t}, \quad \forall k, t$

$\sum_{i=1}^{I} \sum_{k=1}^{K}\left(\frac{y_{i j k t}}{\mathrm{PLC}_{i}}\right) \leqslant \mathrm{CT}_{j t}, \quad \forall j, t$,

$\sum_{j=1}^{J} z_{l h j t} \leqslant \mathrm{CS}_{l h t}, \quad \forall l, h, t$.

Binary constraints:

$$
\begin{aligned}
& x_{i j t} \leqslant G_{i j t}^{x} X_{i j t}, \quad \forall i, j, t, \\
& y_{i j k t} \leqslant G_{i j t}^{y} Y_{i j k t}, \quad \forall i, j, k, t,
\end{aligned}
$$

$$
\begin{aligned}
& u_{i k t} \leqslant G_{i k t}^{u} U_{i k t}, \quad \forall i, k, t, \\
& z_{l h j t} \leqslant G_{l h j t}^{z} Z_{l h j t}, \quad \forall l, h, j, t,
\end{aligned}
$$

$$
\sum_{i=1}^{I} X_{i j t}-1 \leqslant \zeta_{j t}, \quad \forall j, t
$$

Bounds:

$x_{i j t}, y_{i j k t}, u_{i k t}, z_{l h j t}, \operatorname{rm}_{h j t}, I_{i k t}, \tau_{i k t} \geqslant 0, \quad \forall i, j, k, l, h, t$,

$X_{i j t}, Y_{i j k t}, U_{i k t}, Z_{l h j t} \geqslant 0, \quad \forall i, j, k, l, h, t$,

$\zeta_{j t} \geqslant 0$, and integer.

The objective function (1) maximizes the total profit of the supply chain by maximizing the total revenue minus the total costs including the fixed and variable costs of production, raw material procurement and inventory, as well as product transportation, distribution and inventory. Constraints (2a) guarantee a minimum accumulated demand fill rate of $95 \%$ over the whole planning horizon. Constraints (2b) and (2c) ensure the distribution amount equals to demand minus any shortages taking into account inventory level. Constraints (3a) guarantee no inventory at the production sites. Constraints (3b) maintain a sufficient raw material supply over the whole planning horizon. Constraints (3c) and (3d) ensure that sufficient raw materials are available in each period. Constraints (4) ensure that the production amount is greater than a minimum batch size limitation. Constraints $(5 a)-(5 d)$ are a group of capacity limitations including supply, production, distribution and final product transportation. Constraints (6a)-(6d) are binary constraints, (6e) are the general integer constraints for changeover variables. Constraints $(7 a)-(7 c)$ define the variable bounds.

\section{Solution procedure}

The use of conventional tools for solving the MIP problem is limited due to the complexity of the problem and the large number of variables and constraints, particularly for realistically sized problems. Indeed, even for the simpler models studied in the literature, heuristic algorithms have been applied extensively. For example, Jayaraman and Pirkul (2001) outlined a heuristic solution procedure that utilizes the linear programming technique as 
well as results from a Lagrangean relaxation procedure. However, there is no standard heuristic algorithm construction framework for diverse mathematical programming problems. A heuristic procedure is often problem or decision objective oriented. With regard to the MIP model P described above, two specific heuristic algorithms are presented in Sections 4.2 and 4.3 below to solve the problem within an acceptable computation time and without too much loss of optimality. Before we present the two heuristic algorithms, in subsection 3.4.1, the integrated Lagrangian relaxation method is first used to obtain upper bound on the objective value of the original problems. The upper bound will be used to assess the quality of the heuristic solutions.

\subsection{Upper bound}

Lagrangean relaxation is a tool that is increasingly being used in large-scale mathematical programming applications (Fisher, 1985). It provides an upper bound of the objective value for an original maximizing problem. The Lagrangean relaxation method works by relaxing the constraints that cause the computational difficulty, and adding them into the objective function with corresponding Lagrangean multipliers. On the model P, computation experiments relaxing the binary constraints and the capacity constraints, respectively, have been tried; the results indicate that relaxing capacity constraints gave the better solutions. Thus, the algorithm will be constructed by relaxing all capacity constraints (5a)-(5d), and an integrated Lagrangean relaxation model LIP is formulated below

$$
\begin{aligned}
& \max V^{*}= \mathrm{V} \\
&+\sum_{j=1}^{J} \alpha_{j t}\left(\sum_{i=1}^{I}\left(\mathrm{pt}_{i j} x_{i j t}+\mathrm{sut}_{i j} X_{i j t}\right)-\mathrm{CP}_{j t}\right) \\
&+\sum_{k=1}^{K} \beta_{k t}\left(\sum_{i=1}^{I} \mathrm{ups}_{i k} u_{i k t}-\mathrm{CD}_{k t}\right) \\
&+\sum_{j=1}^{J} \lambda_{j t}\left(\sum_{i=1}^{I} \sum_{k=1}^{K} \frac{y_{i j k t}}{\mathrm{PLC}_{i}}-\mathrm{CT}_{j t}\right) \\
&+\sum_{l=1}^{L} \sum_{h=1}^{H} \gamma_{l h t}\left(\sum_{j=1}^{J} z_{l h j t}-\mathrm{CS}_{l h t}\right)
\end{aligned}
$$

Subject to (2a)-(2c), (3a)-(3d), (4), (6a)-(6e), (7a)-(7c), where $\alpha_{j t} \geqslant 0, \beta_{k t} \geqslant 0, \lambda_{j t} \geqslant 0, \gamma_{l h t} \geqslant 0$ are Lagrangean multipliers corresponding to the original constraints (5a)-(5d). In addition, $\mathrm{V}$ is the objective function from equation (1).

The relaxations reduce the numbers of constraints dramatically. Thus, even very large problems can be solved effectively. However, to determine the optimal Lagrangean multiplier is often a difficult task. Subgradient optimization method is often effective to obtain a reasonably good set of multipliers (Larsson et al., 1996), and therefore used here. The steps of the algorithm, numbered 1, are described below (refer to Tragantalerngsak et al., 1997).

Step 1.1: Initialize the Problem LIP with Lagrangean multipliers $\alpha_{j t}, \beta_{k t}, \lambda_{j t}, \gamma_{l h t}=0$.

Step 1.2: Solve the Lagrangean problem LIP using CPLEX and find a feasible solution.

Step 1.3: Calculate subgradients for each relaxed constraints. The subgradients of production capacity are $s p=\left\{s_{j t} \mid s_{j t}=\sum_{i=1}^{I} x_{i j t} p t_{j t}+\sum_{i=1}^{I} X_{i j t}\right.$ sut $_{j t}-$ $\left.\mathrm{CP}_{j t}\right\}$. Similarly, find the transportation capacity, distribution capacity and supply capacity subgradients $s t, s d$, ss.

Step 1.4: If any one of the following terms holds (1) $|s p|^{2}+|s t|^{2}+|s d|^{2}+|s s|^{2}=0$; (2) multipliers are no longer changing; (3) the number of iterations is over 200, stop, and then output the objective value $V^{*}$ and variable solutions. Otherwise, go to Step 1.6.

Step 1.5: Computation of step size $\theta=\rho\left(Z_{\mathrm{UB}}-\right.$ $\left.Z_{\mathrm{LB}}\right) / \sum_{t=1}^{T} \sum_{j=1}^{J} s_{j t}^{2}, \rho=2$.where $Z_{\mathrm{UB}}$ is an estimated value which is greater than the optimal objective value, and $Z_{\mathrm{LB}}$ is the current objective value of LIP from Step 1.2.

Step 1.6: Update the Lagrangean multipliers. Let

$\alpha_{j t}= \begin{cases}\alpha_{j t}+s_{j t}^{*} \theta & \text { if } s_{j t}>0, \\ 0 & \text { if } s_{j t} \leqslant 0 .\end{cases}$

Similarly, update $\beta_{k t}, \lambda_{j t}, \gamma_{l h t}$, then go to Step 1.2.

Algorithm 1 always gives a same or better upper bound than the exact relaxation solutions. However, since the Lagrangean algorithm cannot guarantee the solution to be feasible for the original problem. Therefore, usually, a complete Lagrangean relaxation algorithm should include a step to adjust the solutions to be feasible. However, given the concern of computational complexity, and the large number of the Lagrangean multipliers in problem LIP it is impossible to adjust the final solutions. Thus, the solution found using this approach gives an upper bound rather than an optimum solution to the problem. 


\subsection{A decomposition heuristic algorithm based on Lagrangean relaxation}

Since decomposition methods often help split a super large problem into a few relatively small subproblems and reduce the computation complexity dramatically, some algorithms that combine modern heuristics and decomposition methods continue to be effective and prevalent. For example, the decomposition method based on Lagrangean relaxation has been applied successfully to solve a large scale MIP problem (Jayaraman and Pirkul, 2001).

Observing the characteristics of the problem LIP, it is easy to split the model LIP into the following submodels using surrogates and relaxations: Production stage model, distribution stage model and supply stage model below.

\subsubsection{Production stage submodel DMp}

$$
\begin{aligned}
\max \mathrm{Vp}= & \sum_{t=1}^{T}\left(\sum_{i=1}^{I} p_{i t} \sum_{k=1}^{K} d_{i k t}\right. \\
& -\left(\sum_{i=1}^{I} \sum_{j=1}^{J} \operatorname{cvp}_{i j t} x_{i j t}\right. \\
& \left.\left.+\sum_{i=1}^{I} \sum_{j=1}^{J} \operatorname{cfp}_{i j t} X_{i j t}+\sum_{j=1}^{J} \operatorname{cco}_{j t} \zeta_{j t}\right)\right)
\end{aligned}
$$

Subject to

$$
\begin{aligned}
\sum_{t=1}^{n} \sum_{j=1}^{J} x_{i j t} & \geqslant \sum_{t=1}^{n} \sum_{k=1}^{K} d_{i k t} \times 0.95 \\
\forall i, t, \quad n & =1,2, \ldots, T
\end{aligned}
$$

and (4), (5a), (6a), (6e), (7a)-(7c), where constraints (10) are the surrogate constraints of constraints (2a). Here, a $95 \%$ of accumulated demand fill rate is required as a minimum for each product in all distribution centres.

\subsubsection{Distribution stage submodel DMd}

$$
\begin{aligned}
\min \mathrm{Vd}= & \sum_{t=1}^{T}\left(\sum_{i=1}^{I} \sum_{j=1}^{J} \sum_{k=1}^{K} \sum_{i=1}^{I} \sum_{j=1}^{J} \sum_{k=1}^{K} \mathrm{cvt}_{i j k t} y_{i j k t}\right. \\
& +\sum_{i=1}^{I} \sum_{j=1}^{J} \sum_{k=1}^{K} \mathrm{cft}_{i j k t} Y_{i j k t} \\
& +\sum_{i=1}^{I} \sum_{k=1}^{K} \mathrm{cvs}_{i k t} u_{i k t}
\end{aligned}
$$

$$
\begin{aligned}
& +\sum_{i=1}^{I} \sum_{k=1}^{K} \operatorname{cfs}_{i k t} U_{i k t} \\
& +\sum_{i=1}^{I} \sum_{k=1}^{K} \operatorname{civ}_{i k t} I_{i k t} \\
& +\sum_{i=1}^{I} p_{i t} \sum_{k=1}^{K} \tau_{i k t} \\
& +\sum_{i=1}^{I}\left(\mathrm{cs}_{i t}+p_{i t}\right) \sum_{k=1}^{K} \tau_{i k t} \\
& \left.+\sum_{k=1}^{K} \beta_{k t}\left(\sum_{i=1}^{I} \operatorname{ups}_{i k} u_{i k t}-\mathrm{CD}_{k t}\right)\right)
\end{aligned}
$$

Subject to (2b)-(2c), (3a), (3b), (5b), (5c), (6b), (6d), (7a)-(7c).

In this submodel, distribution capacity constraints are relaxed, since distribution capacity constraints are the reason that there are no feasible solutions for the fixed production plan generated from the production stage submodel, while the plant is often in charge of the purchase of transportation capacity. Therefore, relaxing distribution capacity provides the possibility to find a feasible solution for the original MIP model based on the predetermined production plan from production stage submodel.

\subsubsection{Raw material supply submodel DMs:}

$$
\begin{aligned}
\min \mathrm{Vs}= & \sum_{t=1}^{T}\left(\sum_{l=1}^{L} \sum_{h=1}^{H} \sum_{j=1}^{J} \operatorname{cvv}_{l h j t} z_{l h j t}\right. \\
& +\sum_{l=1}^{L} \sum_{h=1}^{H} \sum_{j=1}^{J} \operatorname{cfv}_{l h j t} Z_{l h j t} \\
& \left.+\sum_{h=1}^{H} \sum_{j=1}^{J} \operatorname{rmsc}_{h j t} \mathrm{rm}_{h j t}\right)
\end{aligned}
$$

Subject to (3c)-(3d), (5d), (6c), and (7a)-(7c).

Submodel DMp is a plant production planning model, DMd is a transportation and distribution planning model and DMs is a raw material procurement planning model. According to the propositions of the Lagrangean relaxation method, the profit of the original problem $\mathrm{P}$ will be obtained by solving the submodels individually. Assume that the available best objective value of these submodels are $\operatorname{Vp}, \operatorname{Vd}(\lambda)$ and $\mathrm{Vs}$, respectively. Then, the overall objective value of model $\mathrm{P}$ can be calculated as $V=\mathrm{Vp}-\mathrm{Vd}(\lambda)-\mathrm{Vs}$.

The following algorithm, numbered 2 , is used to solve the decomposition model. 
Step 2.1: Initialize the problem DMp with the necessary parameters, and solve the problem exactly, output the solutions $x_{i j t}$ and objective value Vp;

Step 2.2: Initialize the problem DMd with the necessary input parameters including $x_{i j t}$;

Step 2.3: Initialize the Lagrangean multipliers $\lambda_{j t}$, and solve the problem;

Step 2.4: Apply the subgradient optimization procedure (see above algorithm 1);

Step 2.5: Adjust the solutions to be feasible; If there exists any subgradients $s d_{j t}>0$, transfer the excess production to an other plant or period with $s d_{j t}<0$ and the least distribution costs $\mathrm{cvv}_{i j k t}$, so that $s d_{j t}<0$ for all plants and periods, and output the solutions of objective value and variables;

Step 2.6: Determine the production plan according to the solutions from Step 2.5, output $x_{i j t}$ and calculate the $\mathrm{Vd}$;

Step 2.7: Solve the models DMs exactly. $x_{i j t}$ will be the input data. Output all variable solutions and objective value Vs;

Step 2.8: Calculate the overall profit $V=\mathrm{Vp}$ $-\mathrm{Vd}(\lambda)-\mathrm{Vs}$, Stop.

The solution from algorithm 2 provides a feasible solution to the model $\mathrm{P}$. This also provides a lower bound to the original problem. Although the computational results indicate that the algorithm is efficient, the decomposition algorithm usually loses some objective profit since it ignores the interactions among different operations facilities and periods. Moreover, the performance of Lagrangean relaxation is not always stable, and the solution often fluctuates dramatically given the selection of the initial Lagrangean multipliers and the determination of step size. Additionally, since the decoupled models ignore the interaction among the different operation stages, it often causes unnecessary shortage in some distribution centres, or unnecessary inventory in some other distribution centres. Therefore, an integrated heuristic approach is developed as described below, in order to try to improve the solution further.

\subsection{An integrated heuristic algorithm}

Bradley and Arntzen (1999) suggested that simultaneous consideration of capacity, production, and inventory decisions yields superior financial results. Thus, concerning the original problem $\mathbf{P}$, an integrated heuristic algorithm, numbered 3 , is described below.
Step 3.1: Solve a simplified problem IHA below derived from the original problem.

$$
\begin{aligned}
\max V= & \sum_{t=1}^{T}\left(\sum_{i=1}^{I} p_{i t} \sum_{k=1}^{K}\left(d_{i k t}-\tau_{i k t}\right)\right. \\
& -\left(\sum_{i=1}^{I} \sum_{j=1}^{J} \sum_{k=1}^{K} \mathrm{cvt}_{i j k t} y_{i j k t}\right. \\
& +\sum_{i=1}^{I} \sum_{j=1}^{J} \sum_{k=1}^{K} \mathrm{cft}_{i j k t} Y_{i j k t} \\
& +\sum_{i=1}^{I} \sum_{k=1}^{K} \operatorname{cvs}_{i k t} u_{i k t} \\
& +\sum_{i=1}^{I} \sum_{k=1}^{K} \operatorname{cfs}_{i k t} U_{i k t} \\
& +\sum_{i=1}^{I} \sum_{k=1}^{K} \operatorname{civ}_{i k t} I_{i k t} \\
& \left.\left.+\sum_{i=1}^{I} \operatorname{cs}_{i t} \sum_{k=1}^{K} \tau_{i k t}\right)\right)
\end{aligned}
$$

Subject to

$$
\begin{aligned}
& \sum_{i=1}^{I} \mathrm{pt}_{i j}\left(\sum_{k=1}^{K} y_{i j k t}\right)+\sum_{i=1}^{I} \mathrm{sut}_{i j} \leqslant \mathrm{CP}_{j t}, \quad \forall j, t, \\
& \sum_{t=1}^{n} \sum_{j=1}^{J} \sum_{i=1}^{I}\left(\sum_{k=1}^{K} y_{i j k t} r_{i h}\right) \leqslant \sum_{t=1}^{n} \sum_{l=1}^{L} \mathrm{CS}_{l h t}, \\
& \quad \forall h, \quad n=1, \ldots, T
\end{aligned}
$$

and (2a)-(2c), (5b), (5c), (6b), (6c), and (7a)-(7c). Here, constraints (14) are transformations of the original constraints $(5 \mathrm{a})$, such that set-up time is always considered for each product, in each plant and each period regardless whether or not there is a set-up for product $i$. Constraints (15) are surrogate constraints of (5d) and (3c). Apparently, the problem IHA can be solved easily due to the reduction of constraints and variables. Output solutions for variables $y_{i j k t}, u_{i k t}$,

Step 3.2: Based on the logical constraints (3a), calculate the variables $x_{i j t}=\sum_{k=1}^{K} y_{i j k t}$.

Step 3.3: Judge whether solutions of the variables $x_{i j t}$ satisfy constraints (4) or not. If there exists $x_{i j t}-\mathrm{MBP}_{i} \times X_{i j t}<0$, adjust the $x_{i j t}$ to equal to the minimum production batch. If there are sufficient excess transportation capacity and distribution capacity in plant $j$ and DC $k$, the corresponding $y_{i j k t}$ with the minimum variable distribution costs will be increased and the amount increased equals to 
$\left(x_{i j t}-\mathrm{MBP}_{i} \times X_{i j t}\right)$ for each product, plant and period; otherwise, reduce the $x_{i j t}$ to 0 , and transform the amount of $x_{i j t}$ to the other plant with sufficient excess production and transportation capacity, and the corresponding $y_{i j k t}$ will be adjusted.

Step 3.4: Calculate the distribution, inventory and shortage amount of product $u_{i k t}, I_{i k t}$ and $\tau_{i k t}$ according to the adjusted production and transportation plan.

Step 3.5: Assign raw material procurement according to $x_{i j t}$. Sort variable supply cost $\operatorname{cvv}_{l h j t}$ in ascending order by suppliers for each plant, each raw material item and each period, and then set procurement plan based on the order considering the capacity constraints of suppliers.

Step 3.6: Adjust the raw material procurement plan. Observing the raw material inventory $\mathrm{rm}_{h j t}>0$, if existed $\operatorname{rmsc}_{h j t} \mathrm{rm}_{h j t}>\operatorname{cfv}_{l h j(t+1)}$ and supplier $l$ still has excess capacity on raw material $h$ in period $t$, transit the stock to be ordered in next period.

Step 3.7: Calculate the total profit and output the solutions on all variables, stop.

\section{Computational study}

The two heuristic algorithms were coded in $\mathrm{VC}++$.net and compared using some test problems categorized into three sizes of small, medium and large. The problem features are described in Table 1.

In practice, some cost and other data can be collected from the real operations, and others could be estimated by practitioners. In this paper, we investigate the general performance of the model and algorithms, and thus, a large quantity of hypothetical data are applied. For example, demand data in each period and each distribution centre are generated from a uniform distribution $U(15,25)$. Capacity of production facility, $\mathrm{CP}_{j t}$, is generated from a uniform distribution $U(p c 1, p c 2)$ where $p c 1$ and $p c 2$ are estimated according to the total demand of all products in all distribution centres in each period and product processing time and set-up time. Similarly, other capacities are generated by corresponding uniform distributions such as $\mathrm{CD}_{k t} \sim$ $U(d c 1, d c 2), \mathrm{CT}_{j t} \sim U(t c 1, t c 2)$ and $\mathrm{CS}_{l h t} \sim U(s c 1$, $s c 2$ ). All costs and other parameters such as unit processing and set-up time are also generated from a series of uniform distribution functions. Finally, the price parameters are generated based on the total cost, including costs for raw materials procurement, product production as well as transportation and distribution. The prices for each product in each period are generated from a uniform distribution function $p_{i t} \sim U(p l, p h) .(p l, p h)$ is a possible price interval of a product estimated based on the total cost from raw material consumption to production, transportation and distribution. For the sake of brevity, the real values of the parameters are omitted.

Tests are implemented first on a PC with a Pentium IV processor and solved using CPLEX9.1. The results indicated that CPLEX performs well for small size problems. However, for medium or large size problems, it often took excessive time and

Table 1

The configuration and scale features of example problems

\begin{tabular}{|c|c|c|c|c|c|c|c|c|c|}
\hline Problem & $\begin{array}{l}\text { No. of } \\
\text { products }\end{array}$ & $\begin{array}{l}\text { No. of } \\
\text { plants }\end{array}$ & $\begin{array}{l}\text { No. of } \\
\text { suppliers }\end{array}$ & $\begin{array}{l}\text { No. of } \\
\text { DCs }\end{array}$ & $\begin{array}{l}\text { No. of } \\
\text { periods }\end{array}$ & $\begin{array}{l}\text { No. of raw } \\
\text { materials }\end{array}$ & $\begin{array}{l}\text { No. of } \\
\text { constraints }\end{array}$ & $\begin{array}{l}\text { No. of } \\
\text { variables }\end{array}$ & $\begin{array}{l}\text { No. of integer } \\
\text { variables }\end{array}$ \\
\hline $\mathrm{S} 1$ & 2 & 3 & 3 & 3 & 4 & 2 & 608 & 721 & 336 \\
\hline $\mathrm{S} 2$ & 4 & 3 & 3 & 3 & 4 & 2 & 928 & 1137 & 528 \\
\hline S3 & 2 & 5 & 4 & 4 & 4 & 4 & 1348 & 1777 & 832 \\
\hline S4 & 4 & 5 & 4 & 4 & 4 & 4 & 1852 & 2473 & 1160 \\
\hline M1 & 10 & 3 & 3 & 3 & 4 & 2 & 1888 & 2385 & 1104 \\
\hline M2 & 20 & 3 & 3 & 3 & 4 & 2 & 3488 & 4465 & 2064 \\
\hline M3 & 10 & 5 & 4 & 4 & 4 & 4 & 3364 & 4561 & 2144 \\
\hline M4 & 20 & 5 & 4 & 4 & 4 & 4 & 5884 & 8041 & 3784 \\
\hline $\mathrm{L} 1$ & 50 & 3 & 3 & 3 & 4 & 2 & 8288 & 10705 & 4944 \\
\hline L2 & 100 & 3 & 3 & 3 & 4 & 2 & 16288 & 21105 & 9744 \\
\hline L3 & 50 & 5 & 4 & 4 & 4 & 4 & 13444 & 18481 & 8704 \\
\hline L4 & 100 & 5 & 4 & 4 & 4 & 4 & 26044 & 35881 & 16904 \\
\hline
\end{tabular}


Table 2

Solution comparison of integrated heuristic algorithm and decomposition heuristic algorithm

\begin{tabular}{|c|c|c|c|c|c|c|c|c|c|c|}
\hline Problems & $\begin{array}{l}\text { Upper } \\
\text { bounds } \\
\text { (1) }\end{array}$ & $\begin{array}{l}\text { Optimal } \\
\text { profit } \\
\text { (2) }\end{array}$ & $\begin{array}{l}\text { CPU } \\
\text { time (s) }\end{array}$ & $\begin{array}{l}\text { Decomposition } \\
\text { heuristic profit } \\
\text { (3) }\end{array}$ & $\begin{array}{l}\text { Gap } 1^{\mathrm{a}} \\
(\%)(4) \\
((1)-(3)) / \\
(1)\end{array}$ & $\begin{array}{l}\text { Gap } 2^{\mathrm{b}} \\
(\%)(5) \\
((2)-(3)) / \\
(2)\end{array}$ & $\begin{array}{l}\text { Integrated } \\
\text { heuristics } \\
\text { profit } \\
\text { (6) }\end{array}$ & $\begin{array}{l}\text { Gap } 3^{\mathrm{c}} \\
(\%)(7) \\
(1)-(6)) / \\
(1)\end{array}$ & $\begin{array}{l}\text { Gap } 4^{\mathrm{d}} \\
(\%)(8) \\
((2)-(6)) / \\
(2)\end{array}$ & $\begin{array}{l}\text { Gap5 } 5^{\mathrm{e}} \\
(\%)(9) \\
((6)-(3)) / \\
(6)\end{array}$ \\
\hline $\mathrm{S} 1$ & 1194260 & 1193220 & 65 & 1178754 & 1.30 & 1.21 & 1192764 & 0.13 & 0.04 & 1.17 \\
\hline $\mathrm{S} 2$ & 2548220 & 2545390 & 21 & 2516649 & 1.24 & 1.13 & 2542940 & 0.21 & 0.10 & 1.03 \\
\hline S3 & 1663270 & 1659640 & 417 & 1639011 & 1.46 & 1.24 & 1658303 & 0.30 & 0.08 & 1.16 \\
\hline S4 & 3172980 & 3162970 & 1736 & 3128332 & 1.41 & 1.10 & 3159566 & 0.42 & 0.11 & 0.99 \\
\hline M1 & 6546090 & & & 6418958 & 1.94 & & 6521420 & 0.38 & & 1.57 \\
\hline M2 & 13344400 & & & 13115624 & 1.71 & & 13287365 & 0.43 & & 1.29 \\
\hline M3 & 8640430 & & & 8509040 & 1.52 & & 8602061 & 0.44 & & 1.08 \\
\hline M4 & 17364000 & & & 17025814 & 1.95 & & 17259748 & 0.60 & & 1.36 \\
\hline L1 & 33298900 & & & 32718096 & 1.74 & & 33205314 & 0.28 & & 1.47 \\
\hline $\mathrm{L} 2$ & 60511400 & & & 59452193 & 1.75 & & 60228230 & 0.47 & & 1.29 \\
\hline L3 & 39776600 & & & 39006021 & 1.94 & & 39598837 & 0.45 & & 1.50 \\
\hline L4 & 79013000 & & & 77390680 & 2.05 & & 78724552 & 0.37 & & 1.69 \\
\hline Average & & & & & 1.67 & 1.17 & & 0.37 & 0.08 & 1.30 \\
\hline
\end{tabular}

${ }^{\mathrm{a}}$ The error rate between the decomposition solution and the upper bound.

${ }^{\mathrm{b}}$ The error rate between the decomposition solution and the optimal value.

${ }^{\mathrm{c}}$ The error rate between the integrated solution and the upper bound.

${ }^{\mathrm{d}}$ The error rate between the integrated solution and the optimal solution.

${ }^{\mathrm{e}}$ The profit increase rate of the integrated algorithm compared with the decomposition algorithm.

sometimes ran out of memory. Therefore, the two heuristic algorithms described in Sections 4.2 and 4.3 are applied and compared below. In addition, upper bounds of the objective value are also calculated by using the integrated Lagrangean relaxation method for all problems.

Firstly, the two heuristic algorithms were used to solve the small size test problems, and the results were compared with those obtained by CPLEX and the upper bounds of the objective value. The detailed computational results are presented in Table 2. The computation indicates that CPLEX solves the problems optimally, but the computation is time consuming, even for these small size problems. However, the decomposition heuristic algorithm and the integrated heuristic algorithm solve the problems within a very short CPU time. Moreover, the integrated solutions are always better than the decomposition ones. The average differences between the two kinds of heuristic solutions and optimal solution are $1.17 \%$ and $0.08 \%$, respectively.

Furthermore, the medium and large problems were solved using the decomposition and integrated heuristic algorithm, respectively, and the results are presented in Table 2 above. If we compare the solutions of the heuristic algorithms with the upper bounds, the average gap is just $1.67 \%$ and $0.37 \%$. Therefore, the solution results of both heuristics are acceptable within a short computational time.

However, the integrated algorithm usually gives a better objective value than the decomposition approach. Observing the maximum profits of the decomposition and integrated heuristics, we found the average gap between them is $1.30 \%$. The integrated algorithm is more flexible to adjust the operations plan than the decomposition algorithm. Moreover, the decomposition solutions often include larger shortages and inventories, and result in the profit decreasing dramatically. In contrast, the integrated heuristic algorithm limits the shortage to be less than $5 \%$.

\section{Sensitivity analysis}

We investigate the robustness of solutions on different capacity levels. Additionally, the price affects revenue significantly, thereby affecting the capacity allocation. Thus, a sensitivity analysis 
considering capacity factors and price factors is carried out.

\subsection{The sensitivity analysis on capacity}

Firstly, with respect to the individual capacities, we solve the problems using CPLEX and the other two heuristic algorithms considering different capacity levels. Each capacity varies from $80 \%$ of the original value to infinite/excess capacity. Then, the gaps between the integrated heuristic solutions and the CPLEX optimal solutions are calculated for small sized problems. Furthermore, the average gap for each kind of capacity is described below as the "average error rate". The results indicate that the integrated heuristic algorithm always provides good solutions with very low average error rate under the different capacity levels (see Fig. 2).

Secondly, the gaps between the decomposition heuristic and integrated heuristic solutions are calculated, and the average gap for all test problems on each type of capacity is denoted below as "average profit increase rate". The variation is presented in Fig. 3. The analysis showed that the integrated heuristic algorithm always provides better solutions than decomposition algorithm does under each capacity level on each individual capacity.

The analysis also indicates that the different algorithms have their special advantages. For example, (1) for the CPLEX solution, the tighter the capacity constraints are, the longer the computation time is; (2) the decomposition algorithm usually allows slightly tighter capacity constraints than the integrated heuristics does by sacrificing some demand fill rate; and (3) the integrated

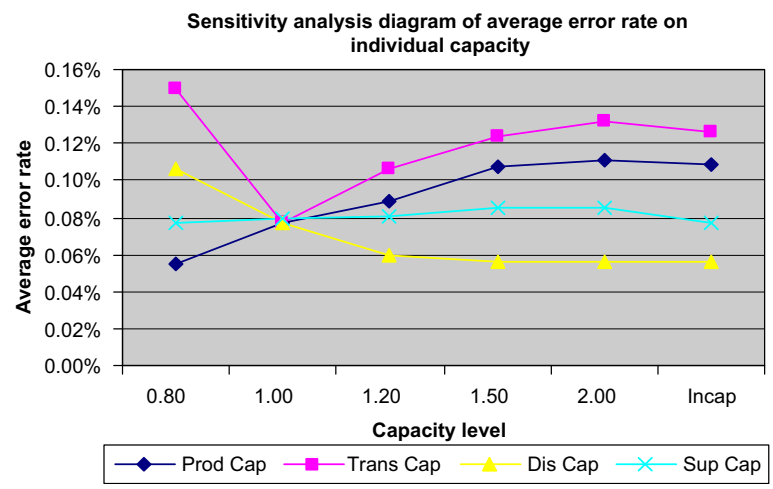

Fig. 2. The integrated heuristic error rate fluctuations based on the variation of capacities.

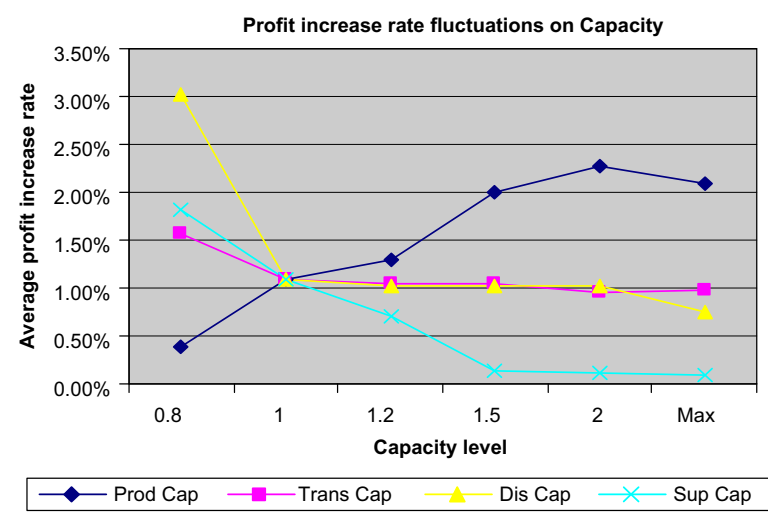

Fig. 3. A comparison of the decomposition and integrated heuristics for different capacity levels.

heuristic algorithm is more time consuming than decomposition heuristics, especially to the very large problem.

\subsection{The sensitivity analysis on price}

Since the price always impacts the profit significantly, it is necessary to discuss the influence of price on the solutions of the model for the decomposition heuristic algorithm and the integrated heuristic algorithm. Here, we solved the test problems in Table 1 with six different price intervals from the lowest price level interval $[1000,1500]$ to the highest price level interval of $[1500,2000]$, and calculate the average profit increase rates, where the profit increase rate is the ratio of the difference between the decomposed profit and the integrated profit and the integrated profit for each instance. The average profit increase rates are the average values of the profit increase rate of the all instances at each price level.

The results indicate that all solutions from different algorithms still satisfy the relationship found in the Section 5, that is, the integrated algorithm gave better solutions than the decomposition algorithm. In addition, the profit increase rate between the integrated heuristics and the decomposition heuristics will decrease with the increase of the price (see Fig. 4). In such a complicated model, many reasons could cause this result. At this stage, we only present the numerical results, and did not address the reasons in detail. Potentially, this might be caused by different shortage levels in the decomposition solutions and the integrated solutions at the same price. For 
example, at a lower price, the decomposition algorithm solution is more likely to include a shortage than the integrated algorithm since the price level only impacts the production stage decision. With the increase of the price, both algorithms opt to satisfy demands completely due to the higher profit margin.

Although the average profit increase rate increases with the reduction of the price, it continues to be between $1 \%$ and $5 \%$. With such a large and complicated mathematical model, it is likely that an even larger gap of $5 \%$ would be acceptable in practice. Therefore, it is concluded that both algorithms give good solutions although the integrated heuristic always performs better.

\section{Comparison with adapted version of Park's model}

Park studied an integrated production and distribution planning problem without the consideration of raw material procurement activities. He tested the approach using a number of hypothetical

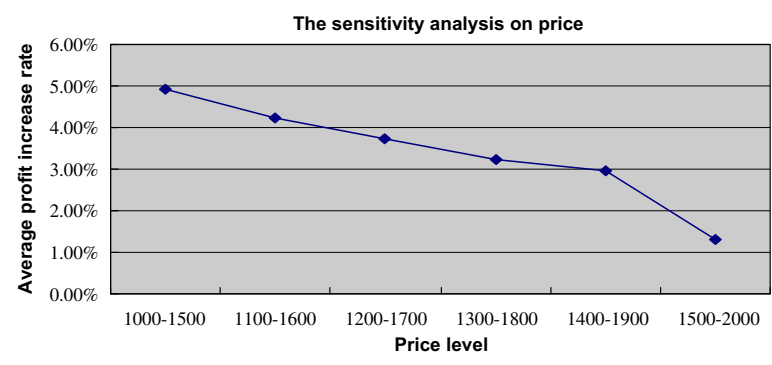

Fig. 4. A comparison of the decomposition and integrated heuristics for different price levels. test problems. We adapted those test problems, supplemented with two suppliers and two types of raw material, to give seven new small sized instances, S1-S7 (see Table 3 for the instance features).

The purpose of the comparison study is to verify that the integrated solutions considering the whole supply chain from raw material supply to final product distribution are always better than the solutions from solving the production-distribution problem and raw material procurement separately. The two stage decoupled model is reformulated by taking the relevant supply activities out of the model $\mathrm{P}$ to fit Park's problem network, called production distribution submodel and raw material procurement submodel.

For the test problems, both the integrated solution and the decoupled solutions are found using Cplex. For two instances, S5 and S7, the decoupled solutions are not feasible. It turns out that for these instances, the production-distribution part of the solution determined cannot be achieved by the raw material procurement submodel because of material shortages. To be able to judge the quality of the decoupled solution aside from this material shortage, we solved the raw material procurement submodel with sufficient material available. The profits for the integrated solution and the decoupled solutions are compared in Table 4.

The results in Table 4 show that the optimal integrated solution is always better than the optimal decoupled solution, even when we allow additional capacity in the decoupled approach to obtain a feasible solution.

Table 3

The structure of the test problems

\begin{tabular}{|c|c|c|c|c|c|c|}
\hline \multirow[t]{3}{*}{ Problem } & \multicolumn{6}{|c|}{ Integrated capacity allocation for complex supply chain } \\
\hline & \multicolumn{4}{|c|}{ Production-distribution planning } & \multicolumn{2}{|l|}{ Supply planning } \\
\hline & Product item & No. of plants & No. of DCs & No. of periods & No. of suppliers & Raw material item \\
\hline $\mathrm{S} 1$ & 2 & 2 & 5 & 5 & 2 & 2 \\
\hline $\mathrm{S} 2$ & 3 & 2 & 5 & 5 & 2 & 2 \\
\hline S3 & 2 & 2 & 8 & 5 & 2 & 2 \\
\hline S4 & 2 & 2 & 10 & 5 & 2 & 2 \\
\hline S5 & 3 & 2 & 8 & 5 & 2 & 2 \\
\hline S6 & 2 & 2 & 12 & 5 & 2 & 2 \\
\hline S7 & 3 & 2 & 10 & 5 & 2 & 2 \\
\hline
\end{tabular}


Table 4

Comparison of exact solutions for the integrated and decoupled approach for small size problems

\begin{tabular}{|c|c|c|c|c|c|}
\hline \multirow{2}{*}{ Problems } & \multirow{2}{*}{$\begin{array}{l}\text { Integrated profit } \\
\text { A }\end{array}$} & \multicolumn{3}{|l|}{ Decoupled } & \multirow[t]{2}{*}{$\operatorname{Gap}(\mathrm{D}-\mathrm{A}) / \mathrm{A}(\%)$} \\
\hline & & $\begin{array}{l}\text { Prod-Dis profit } \\
\text { B }\end{array}$ & $\begin{array}{l}\text { Sup cost } \\
\text { C }\end{array}$ & $\begin{array}{l}\text { Total profit } \\
\mathrm{D}=\mathrm{B}-\mathrm{C}\end{array}$ & \\
\hline S1 & 1671860 & 1685090 & 49274 & 1635816 & 2.16 \\
\hline $\mathrm{S} 2$ & 2415610 & 2458750 & 73868 & 2384882 & 1.27 \\
\hline S3 & 2668710 & 2661200 & 90358 & 2570842 & 3.67 \\
\hline S4 & 3443740 & 3364510 & 71207 & 3293303 & 4.37 \\
\hline S5 & 3851780 & 3868060 & Infeasible & $\mathrm{N} / \mathrm{A}$ & $\mathrm{N} / \mathrm{A}$ \\
\hline S5\# & 3853720 & 3868060 & 152236 & 3715824 & 3.58 \\
\hline S6 & 3995360 & 3879830 & 103987 & 3775843 & 5.49 \\
\hline S7 & 4851850 & 4867760 & Infeasible & $\mathrm{N} / \mathrm{A}$ & $\mathrm{N} / \mathrm{A}$ \\
\hline S7\# & 4956550 & 4867760 & 130260 & 4737500 & 4.42 \\
\hline
\end{tabular}

We also tested a number of medium and large sized instances, using a similar method to that used for the small sized problems. The results indicated that Cplex is no longer effective, even for the production-distribution planning submodel of the decoupled approach. Instead, we determine an integrated solution using the integrated heuristic (see Section 4.3) algorithm. However, using this method, the decoupled solution is infeasible for most problems. Hence, no comparison could be made and the results are omitted.

\section{Conclusions}

Strategic supply chain planning research for real world problems continues to be an active research area. This study solves a complicated integrated capacity allocation problem for a complicated supply chain. Multiple capacity constraint factors and fixed costs are taken into account in the model. The correspondent heuristic algorithms are efficient and give very good solutions within an acceptable computation time and with an acceptable error rate. A comparison research between this study and an adapted version of Park's model is carried out. It indicates that the integrated planning approach for a complete supply chain always presents better solution than the decoupled planning method. Future research also could extend the model and algorithms to consider the multi-production line in all plants. Furthermore, capacity expansion decisions could be considered. In addition, demand uncertainty is an interesting topic which warrants further study in this context.

\section{References}

Bradley, J.R., Arntzen, B.C., 1999. The simultaneous planning of production, capacity, and inventory in seasonal demand environments. Operations Research 47, 795-806.

Chen, M., Wang, W., 1997. A linear programming model for integrated steel production and distribution planning. International Journal of Production Management 17, 592-610.

Dhaenens-Flipo, C., Finke, G., 2001. An integrated model for an industrial production-distribution problem. IIE Transactions 33, 705-715.

Eppen, G.D., Martin, R.K., Schrage, L., 1989. A scenario approach to capacity allocation. Operations Research 37, 517-527.

Fisher, M.L., 1985. An applications oriented guide to Lagrangean relaxation. Interfaces $15,10-21$.

Jayaraman, V., Pirkul, H., 2001. Planning and coordination of production and distribution facilities for multiple commodities. European Journal of Operational Research 133, 394-408.

Larsson, T., Patriksson, M., Stromberg, A.-B., 1996. Conditional subgradient optimization-theory and applications. European Journal of Operational Research 88, 382-403.

Lim, S.K., Kim, Y.D., 1999. An integrated approach to dynamic plant location and capacity allocation. Journal of Operational Research 50, 1205-1216.

Park, Y., 2005. An integrated approach for production and distribution planning in supply chain management. International Journal of Production Research 43, 1205-1224.

Ryu, J.H., Dua, V., Pistikopoulos, E.N., 2004. A bilevel programming framework for enterprise-wide process networks under uncertainty. Computers and Chemical Engineering 28, 1121-1129.

Tragantalerngsak, S., Holt, J., Ronnqvist, M., 1997. Lagrangean heuristics for the two-echelon, single-source, capacitated facility location problem. European Journal of Operational Research 102, 611-625.

Vidal, C.J., Goetschackx, M., 1997. Strategic production distribution models: A critical review with emphasis on global supply chain models. European Journal of Operational Research 98, 1-18. 